\title{
Electron Fractionalization in Two-Dimensional Graphenelike Structures
}

\author{
Chang-Yu Hou, ${ }^{1}$ Claudio Chamon, ${ }^{1}$ and Christopher Mudry ${ }^{2}$ \\ ${ }^{1}$ Physics Department, Boston University, 590 Commonwealth Avenue, Boston, Massachusetts 02215, USA \\ ${ }^{2}$ Condensed Matter Theory Group, Paul Scherrer Institut, CH-5232 Villigen PSI, Switzerland
}

(Received 28 September 2006; published 4 May 2007)

\begin{abstract}
Electron fractionalization is intimately related to topology. In one-dimensional systems, fractionally charged states exist at domain walls between degenerate vacua. In two-dimensional systems, fractionalization exists in quantum Hall fluids, where time-reversal symmetry is broken by a large external magnetic field. Recently, there has been a tremendous effort in the search for examples of fractionalization in twodimensional systems with time-reversal symmetry. In this Letter, we show that fractionally charged topological excitations exist on graphenelike structures, where quasiparticles are described by two flavors of Dirac fermions and time-reversal symmetry is respected. The topological zero modes are mathematically similar to fractional vortices in $p$-wave superconductors. They correspond to a twist in the phase in the mass of the Dirac fermions, akin to cosmic strings in particle physics.
\end{abstract}

DOI: $10.1103 /$ PhysRevLett.98.186809

In low-dimensional systems, the excitation spectrum sometimes contains quasiparticles with fractionalized quantum numbers. A famous example of fractionalization was obtained in one dimension (1D) by Jackiw and Rebbi [1] and by Su, Schrieffer, and Hegger [2]. They showed the existence of charge $e / 2$ states, with polyacetelene as a physical realization of such phenomena. In these systems, a charge density wave develops and the ground state is twofold degenerate. The fractionalized states correspond to midgap or zero-mode solutions that are sustained at the domain wall (a soliton) interpolating between the twodegenerate vacua.

The fractional quantum Hall effect provides an example of fractionalization in two dimensions (2D). Not only do the Laughlin quasiparticles have fractional charge [3], but they also have fractional (anyon) statistics [4,5]. Timereversal symmetry (TRS) is broken due to the strong magnetic field, leaving as an outstanding problem the search for systems where fractionalization is realized without the breaking of TRS. The motivation for such a quest stems from speculations that fractionalization may play a role in the mechanism for high-temperature superconductivity [6-8]. Progress has been made on finding model systems, such as dimer models, in which monomers' defects act as fractionalized (and deconfined, in the case of the triangular lattice) excitations.

In this Letter, we present a mechanism to fractionalize the electron in graphenelike systems that leaves TRS unbroken. The excitation spectrum of honeycomb lattices, which have been known theoretically for a few decades to be described by Dirac fermions $[9,10]$, is now the subject of many recent studies since single and few atomic-layer graphite samples have been realized experimentally [11]. Quantum number fractionalization is intimately related to topology and here we find that a twist or a vortex in an order parameter for a mass gap gives rise to a single midgap state at zero energy. Such twist in the mass of the Dirac fermions in graphenelike structure is the analogous
PACS numbers: 73.20.Mf, 05.30.Pr, 71.10.Fd, 71.23.An

in $2+1$ space-time dimensions of a cosmic string in $3+1$ dimensions [12].

The zero modes we find are, in their mathematical structure, similar to those found in $p$-wave superconductors by Read and Green [13] and to those found by Jackiw and Rossi [14] when demonstrating topological excitations and suggesting an index theorem in 2D (see also Ref. [15]). In Refs. [13-15], a twist in the phase of a superconducting order parameter and a charge $2 e$ Higgs boson, respectively, were considered and thus the electric charge assigned to the zero-mode is not a good fractional quantum number. Instead, in the systems we study, the electronic charge is conserved and therefore we can show that it is fractionalized by properly tallying it.

We find only one normalizable state for each vortex, despite the two flavors of Dirac fermions in honeycomb lattices. The fact that there is one and not two zero modes is essential for fractionalization: doubling the number of zero modes due to two flavors of Dirac fermions, in addition to the spin degeneracy, would lead to excitations with the same quantum numbers as ordinary electrons. We find excitations with charge $Q=e / 2$ in the spinless case, and charge $Q=e$ and spin $S=0$ or charge $Q=0$ and spin $S=1 / 2$ in the case with spin. Hence, these fractionalized quantum numbers are similar to those in polyacetelene, but the counting is different in $1 \mathrm{D}$ and $2 \mathrm{D}$, and as we will discuss, there is a $U(1)$ symmetry underlying the fractionalization in the 2D case which would not lead to fractionalization in $1 \mathrm{D}$ (in which a $\mathbb{Z}_{2}$ symmetry is underlying the effect).

The mechanism for fractionalization can be portrayed in a simple form by considering spinless electrons hopping on a honeycomb lattice with textured tight-binding hopping amplitudes and described by the Hamiltonian

$$
H=-\sum_{r \in \Lambda_{A}} \sum_{i=1}^{3}\left(t+\delta t_{\mathbf{r}, i}\right) a_{r}^{\dagger} b_{r+s_{i}}+\text { H.c. }
$$


Here, $\boldsymbol{s}_{i}$ with $i=1,2,3$ connects any site $\boldsymbol{r}$ belonging to the triangular sublattice $\Lambda_{A}$ to its three nearest neighbors belonging to the triangular sublattice $\Lambda_{B}$ of the honeycomb lattice as is depicted in Fig. 1. The fermionic annihilation operators $a_{\boldsymbol{r}}$ and $b_{\boldsymbol{r}}$ act on $\Lambda_{A}$ and $\Lambda_{B}$, respectively, and so do their adjoints. Graphene is often described by Hamiltonian (1) in the single-particle approximation, neglecting the spin of the electron and assuming $\delta t_{r, i}=0$. We are going to show that the small variations of the hopping strength, $\delta t_{r, i}$, over the uniform hopping $t$ provide the background on which fractionally charged states can be constructed. We then discuss how such $\delta t_{r, i}$ can arise from a local order parameter that decouples electron-electron interactions.

When $\delta t_{r, i}=0$, Hamiltonian (1) can be diagonalized in momentum space, $H=\sum_{k} \Phi_{k} a_{k}^{\dagger} b_{k}+$ H.c., $\Phi_{k}=$ $-t \sum_{j=1}^{3} e^{i \boldsymbol{k} \cdot s_{j}}$. The single-particle spectrum $\varepsilon_{k}= \pm\left|\Phi_{k}\right|$ thus contains two (zero-energy) Dirac points at the zone boundaries $\boldsymbol{K}_{ \pm}= \pm\left(\frac{4 \pi}{3 \sqrt{3} a}, 0\right)$. After linearization, $\boldsymbol{k}=\boldsymbol{K}_{ \pm}+\boldsymbol{p}$, one obtains a spectrum containing two chiral flavors,$\pm \mathcal{H}=\sum_{p, \pm} \phi_{p} a_{p, \pm}^{\dagger} b_{p, \pm}+$ H.c., $\phi_{p, \pm}=$ $\pm \boldsymbol{v}_{F}\left(p_{x} \pm i p_{y}\right)$ with the Dirac conelike structure $\varepsilon_{ \pm}(\boldsymbol{p})=$ $\pm v_{F}|p|$ for the energy dispersion. (Hereafter, $v_{F}=1$.)

We shall focus on backgrounds that yield a chiral mixing between the \pm species. A Kekulé texture, depicted in Fig. 1, provides such a mixing [16]. The modulation

$$
\delta t_{\boldsymbol{r}, i}=\Delta(\boldsymbol{r}) e^{i \boldsymbol{K}_{+} \cdot \boldsymbol{s}_{i}} e^{i \boldsymbol{G} \cdot \boldsymbol{r}} / 3+\text { c.c., }
$$

with wave vector $\boldsymbol{G}:=\boldsymbol{K}_{+}-\boldsymbol{K}_{-}$, couples the Dirac points at $\boldsymbol{K}_{ \pm}$as is depicted in Fig. 2. Here, we allow for spatial fluctuations (on length scales much longer than the lattice spacing $a$ ) of the complex-valued order parameter $\Delta(\boldsymbol{r})$. The phase of $\Delta(\boldsymbol{r})$ controls the ordered hopping texture. To leading order in a gradient expansion, Hamiltonian (1) subjected to the Kekule texture (2) is given by $\mathcal{H}=\int d^{2} \boldsymbol{r} \Psi^{\dagger}(\boldsymbol{r}) \mathcal{K}_{D}(\boldsymbol{r}) \Psi(\boldsymbol{r})$ with $\Psi^{\dagger}(\boldsymbol{r})=$

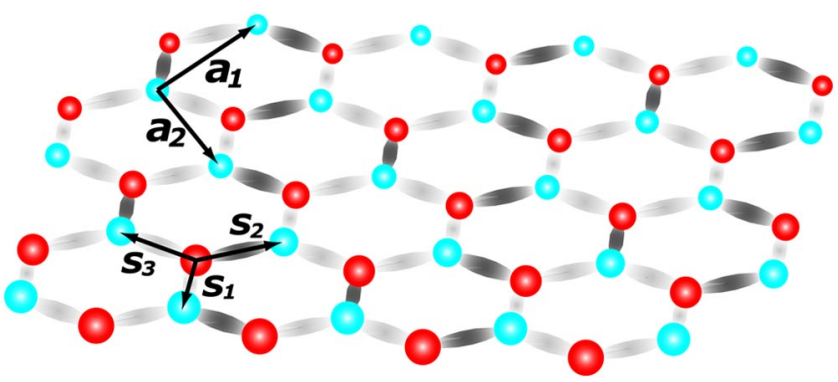

FIG. 1 (color online). The honeycomb lattice $\Lambda$ with lattice spacing $a$ and its two triangular sublattice $\Lambda_{A}$ (red) and $\Lambda_{B}$ (blue). The generators of $\Lambda_{A}$ are $\boldsymbol{a}_{1}$ and $\boldsymbol{a}_{2}$. The three vectors $\boldsymbol{s}_{j}$ connect any site from $\Lambda_{A}$ to its three nearest-neighbor sites belonging to $\Lambda_{B}$. The Kekule distortion is a modulation of the nearest-neighbor hopping amplitude that is indicated by representing nearest-neighbor bonds of the honeycomb lattice in black (gray) if the hopping amplitude is large (small). $\left[u_{b}^{\dagger}(\boldsymbol{r}) u_{a}^{\dagger}(\boldsymbol{r}) v_{a}^{\dagger}(\boldsymbol{r}) v_{b}^{\dagger}(\boldsymbol{r})\right]$ and

$$
\mathcal{K}_{D}=\left(\begin{array}{cccc}
0 & -2 i \partial_{z} & \Delta(\boldsymbol{r}) & 0 \\
-2 i \partial_{\bar{z}} & 0 & 0 & \Delta(\boldsymbol{r}) \\
\bar{\Delta}(\boldsymbol{r}) & 0 & 0 & 2 i \partial_{z} \\
0 & \bar{\Delta}(\boldsymbol{r}) & 2 i \partial_{\bar{z}} & 0
\end{array}\right) .
$$

We are using the notation $z=x+i y, \partial_{z}=\left(\partial_{x}-i \partial_{y}\right) / 2$, with an overline to denote complex conjugation. Without the Kekulé texture (2) leading to $\Delta(\boldsymbol{r})$, the Dirac kernel (3) is the single-particle relativistic massless Dirac Hamiltonian in $2+1$ space-time. With the Kekule texture (2) $\Delta(\boldsymbol{r})=\Delta_{0}$, the dispersion takes the simple form $\varepsilon_{ \pm}(p)= \pm \sqrt{|\boldsymbol{p}|^{2}+\left|\Delta_{0}\right|^{2}}$; i.e., a single-particle mass gap $\left|\Delta_{0}\right|$ has opened. The Dirac kernel (3) is TRS. TRS originates in the tight-binding hopping elements being real. Moreover, the transformation law under $a_{r} \rightarrow-a_{r}$ and $b_{r+s_{i}} \rightarrow+b_{r+s_{i}}$ of the single-particle tight binding (1) ensures that any positive energy eigenstate of the Dirac kernel (3) can be matched to a negative energy eigenstate, while only zero modes can be left unmatched. We shall call this property sublattice symmetry (SLS) [17].

The order parameter $\Delta(\boldsymbol{r})=\Delta_{0}$ can be complex valued, but the spectral mass gap is real. This suggests that the phase of $\Delta_{0}$ is redundant. In fact, it can be removed from Eq. (3) with a chiral transformation that rotates the phases of the \pm species by opposite angles. This is not true anymore if the phase of the order parameter $\Delta(\boldsymbol{r})$ varies in space, and, in particular, if it contains vortices. The latter situation leads to fractionalization.

We assume that

$$
\Delta(\boldsymbol{r})=\Delta_{0}(r) e^{i(\alpha+n \theta)},
$$

where $\Delta_{0}(r)>0, n \in \mathbb{Z}$ for a single-valued order parameter, and we are using the polar coordinates $z=r \exp (i \theta)$. We are seeking eigenstates of the Dirac kernel (3) with vanishing energy that are normalizable, i.e.,

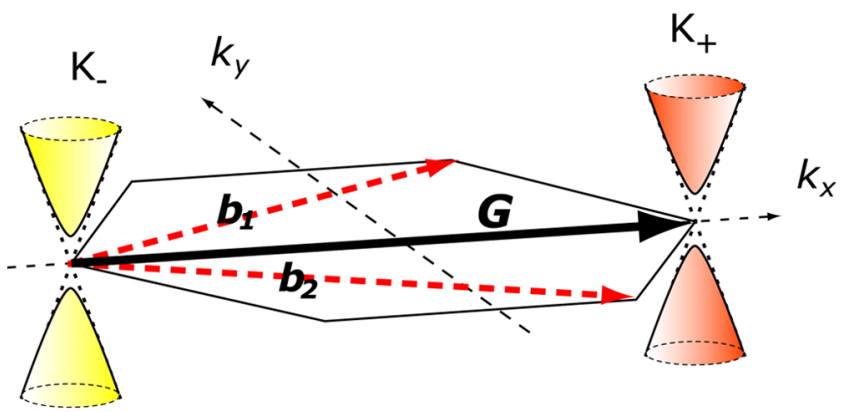

FIG. 2 (color online). The first Brillouin zone of the triangular lattice is shown together with the Dirac points $\boldsymbol{K}_{ \pm}$at the zone boundary, the reciprocal vector $\boldsymbol{G}$ that connects them, and the massive relativistic dispersion centered about $\boldsymbol{K}_{ \pm}$that opens due to a Kekulé distortion with $\Delta(\boldsymbol{r})=\Delta_{0}$. 


$$
\begin{array}{r}
\left(\partial_{r}-i r^{-1} \partial_{\theta}\right) u_{a}(\boldsymbol{r})+i e^{i \theta} \Delta(\mathbf{r}) v_{a}(\boldsymbol{r})=0, \\
i e^{-i \theta} \bar{\Delta}(\boldsymbol{r}) u_{a}(\boldsymbol{r})-\left(\partial_{r}+i r^{-1} \partial_{\theta}\right) v_{a}(\boldsymbol{r})=0,
\end{array}
$$

holds on sublattice $\Lambda_{A}$ while two more equations obtained from Eq. (5) with the substitutions $u_{a} \rightarrow u_{b}, v_{a} \rightarrow v_{b}$, and $\theta \rightarrow-\theta$ must also hold on sublattice $\Lambda_{B}$. The same equations were studied in Refs. [13,14] from a different perspective since the textured gap in these works is associated to a superconducting order parameter. Hence, electric charge is not a conserved quantum number in Refs. [13,14]. There are $|n|$ independent normalizable zero modes, which are either supported on sublattice $\Lambda_{A}$ when $n \leq-1$ or on sublattice $\Lambda_{B}$ when $n \geq 1$.

We assume that $n=-1$ in which case the single-valued and normalizable wave functions for the zero mode is

$$
\begin{aligned}
& u_{a}(r, \theta)=\frac{e^{i[(\alpha / 2)+(\pi / 4)]}}{2 \sqrt{\pi}} \frac{e^{-\int_{0}^{r} d r^{\prime} \Delta_{0}\left(r^{\prime}\right)}}{\sqrt{\int_{0}^{\infty} d r r e^{-2 \int_{0}^{r} d r^{\prime} \Delta_{0}\left(r^{\prime}\right)}}}, \\
& v_{a}(r, \theta)=\bar{u}_{a}(r, \theta) .
\end{aligned}
$$

Its support is on sublattice $\Lambda_{A}$. The zero mode when $n=1$ is obtained from Eq. (7) with the substitutions $u_{a}(r, \theta) \rightarrow$ $v_{b}(r, \theta)$ and $v_{a}(r, \theta) \rightarrow u_{b}(r, \theta)$. When $n=1$, the support of the zero mode is on sublattice $\Lambda_{B}$. The wave function (7) decays exponentially fast away from the core of the vortex (4) at the origin of the complex plane. Its localization length is set by the gap value $\Delta_{0}$ reached when $\Delta_{0}(r)$ saturates sufficiently far from the origin. If the Kekulé texture (2) supports a pair of vortices a large distance $R$ apart, then the Dirac kernel (3) has two eigenstates whose energy eigenvalues are exponentially small.

To obtain the charge bound to a vortex, one has to study $\delta \nu(\boldsymbol{r}, \boldsymbol{\varepsilon}) \equiv \nu_{|n|=1}(\boldsymbol{r}, \boldsymbol{\varepsilon})-\nu_{|n|=0}(\boldsymbol{r}, \boldsymbol{\varepsilon})$, where $\nu_{n}(\boldsymbol{r}, \boldsymbol{\varepsilon})$ is the local density of states (LDOS) of the Dirac kernel (3) in the presence of the mass twist (4). Because of the SLS, to any negative eigenstate of the Dirac kernel, $\psi_{-\varepsilon}(\boldsymbol{r})$, there corresponds a positive energy state, $\psi_{+\varepsilon}(\boldsymbol{r})$, related to $\psi_{-\varepsilon}(\boldsymbol{r})$ by a unitary transformation. Hence, the LDOS $\nu(r, \varepsilon) \equiv$ $\sum_{\varepsilon^{\prime}} \psi_{\varepsilon^{\prime}}^{\dagger}(\boldsymbol{r}) \psi_{\varepsilon^{\prime}}(\boldsymbol{r}) \delta\left(\varepsilon-\varepsilon^{\prime}\right)$ is symmetric with respect to zero energy and negative and positive energy eigenstates contribute equally to the local density $\nu(\boldsymbol{r}) \equiv$ $\lim _{\varepsilon \rightarrow \infty} \int_{-\varepsilon}^{+\varepsilon} d \varepsilon^{\prime} \nu\left(r, \varepsilon^{\prime}\right)$. In the presence of the zero mode $\psi_{0}(\boldsymbol{r})$ this spectral symmetry together with the conservation of the total number of states imply

$$
\int d^{2} \boldsymbol{r}\left[2 \int_{-\infty}^{0^{-}} \delta \nu(\boldsymbol{r}, \varepsilon) d \varepsilon+\left|\psi_{0}(\boldsymbol{r})\right|^{2}\right]=0 .
$$

But the single zero mode $\psi_{0}(\boldsymbol{r})$ is normalized to one and

$$
\int d^{2} \boldsymbol{r} \int_{-\infty}^{0^{-}} \delta \nu(\boldsymbol{r}, \boldsymbol{\varepsilon}) d \boldsymbol{\varepsilon}=-1 / 2 .
$$

According to Eq. (8), the valence band has a deficit of half of a state, as does the conduction band. Thus the difference in net charge between a fully occupied valence band with and without the vortex is $-e / 2$. The total charge of a closed system must still be an integer. This can be understood as follows. Vortices must be present in pairs (for example, if a finite system is to satisfy periodic boundary conditions or for energetic reasons in the thermodynamic limit), and while the charge around each vortex is half that of an electron, the fact that the vortices appear in pairs ensures that the total charge is an integer multiple of $e$. The mechanism for fractionalization is similar to that in 1D [18], but there the fractional charge stands in domain walls between two degenerate vacua, which must appear in pairs (again if the system is to satisfy periodic boundary conditions). In 1D, however, one needs a discrete symmetry to fractionalized charge. For example, for charge $e / 2$ states one needs a $\mathbb{Z}_{2}$ symmetry, which can be understood generically in terms of an accumulation of charge along phase twists that have a natural interpretation within a bosonization scheme [19]. These arguments do not extend to $2 \mathrm{D}$, which is our concern in this Letter, as there is no simple connection between phase twist and charge as in the $1 \mathrm{D}$ bosonization scheme. The fact that the fractional states must occur in pairs, nonetheless, follows from the common requirement for the $U(1)$ mass vortices in $2 \mathrm{D}$ and the $\mathbb{Z}_{2}$ domain walls that these defects occur in pairs to satisfy appropriate boundary conditions.

In the case of electrons with spin, two independent zero modes that carry the spin quantum number are expected. Hence, there is, in total, a full electronic state missing in the valence band for each vortex. Consequently, unoccupied, singly, and doubly occupied zero-energy states correspond to the charge and spin quantum numbers $(Q=-e, S=0),(Q=0, S=1 / 2)$, and $(Q=+e, S=$ $0)$, respectively. Notice that, had we obtained two normalizable solutions for a vortex or an antivortex as opposed of one as in Eq. (6), all the quantum numbers would be "doubled" and simply coincide with those of ordinary electrons.

These arguments for fractionalization require existence of the midgap state and the SLS of the spectrum. SLS is broken when a next-nearest-neighbor hopping $t^{\prime}$ is introduced. A $t^{\prime}$ brings an overall shift and a quadratic term into the diagonal sector of the Hamiltonian, $\delta \mathcal{H}^{\prime}=\left(3 t^{\prime}+\right.$ $\left.\frac{9 t^{\prime} \alpha^{2}}{4} p^{2}\right) \mathbb{1}$, up to second order in $\boldsymbol{p}$. This perturbation alters the energy spectrum, but Dirac points (in the absence of the background) remain when the system is at half filling. The textured background still opens a gap at the Dirac points and there is a midgap state if there is a mass twist. However, the midgap state is not exactly at the center between the valence and conduction bands, being shifted by $\delta \varepsilon^{(1)}=\frac{t^{\prime} \Delta_{0}}{t^{2}} \Delta_{0}$ to first order $t^{\prime}$. As long as this shift is small compared to the gap, the midgap solution is robust against breaking of the SLS. The fractionalized quantum number persists along with the single bound state, but the argument for charge $e / 2$ is not valid. Starting from zero $t^{\prime}$, turning it adiabatically, one can argue that fraction- 
alization should remain but take irrational values as in $1 \mathrm{D}$ [20-22].

We now turn to a mechanism for generating spontaneously the Kekulé distortions from repulsive electronelectron interactions. We assume the nearest-neighbor interaction $H^{\prime}:=-V \sum_{r \in \Lambda_{A}} \sum_{j=1}^{3} a_{r}^{\dagger} b_{r+s_{j}} b_{r+s_{j}}^{\dagger} a_{r}$ with $V$ the interaction strength. Evidently, this interaction preserves TRS, the point-group symmetry of the honeycomb lattice, and SLS (up to the total number operator). After linearization around the unperturbed Dirac points, a mean-field decoupling of this interaction with respect to the order parameter

$$
-\frac{2}{3 V} \Delta=\left\langle b_{-}^{\dagger}\left(\boldsymbol{r}+\boldsymbol{s}_{j}\right) a_{+}(\boldsymbol{r})\right\rangle=\left\langle a_{-}^{\dagger}(\boldsymbol{r}) b_{+}\left(\boldsymbol{r}+\boldsymbol{s}_{j}\right)\right\rangle
$$

coincides with the case of the Kekule distortions. If the system is at half filling and zero temperature, all negative energy states are occupied and the self-consistent equation $1=\frac{3 V}{2\left|\Lambda_{A}\right|} \sum_{p} \frac{\exp \left(i p \cdot s_{j}\right)}{\sqrt{|\boldsymbol{p}|^{2}+|\Delta|^{2}}}$ follows. The sum can be replaced by an integral up to an appropriate momentum cutoff, $\Lambda \mathfrak{a} \sim$ $\frac{2 \pi^{1 / 2}}{3^{3 / 4}}$, here chosen so as to match the total number of states in the effective theory to that in the microscopic theory. As this integral converges even without the $|\Delta|^{2}$ term, neglecting it yields $1 \leq \frac{3 \sqrt{3} V \Lambda \mathfrak{a}}{8|t|}\left[J_{0}(\Lambda \mathfrak{a}) H_{-1}(\Lambda \mathfrak{a})-J_{-1}(\Lambda \mathfrak{a}) \times\right.$ $\left.H_{0}(\Lambda \mathfrak{a})\right]$. Here, $J_{\nu}(z)$ is a Bessel function and $H_{\nu}(z)$ is a Struve function. Insertion of the numerical value for $\Lambda a$ then yields the lower bound $V_{c} \geq \frac{|t|}{0.52465}=1.906|t|$. Selfconsistency demands a repulsive interaction. (The very same mean-field theory also renormalizes the uniform hopping: $t \rightarrow t+\delta t, \delta t \sim 0.1 \mathrm{~V}$.) We note that the condition for the Kekule pattern to form could potentially be engineered in a gas of dipolar fermionic atoms trapped in an optical honeycomb lattice, where the ratio $V / t$ could be tuned.

At last, we need a vortex on top of the Kekule distortion (2) for fractionalization to happen. Here, we face a difficulty in that the chiral symmetry of Eq. (3) is an artifact of the linearization. Integration of the fermions on the lattice yields an effective action for the phase of $\Delta$ with a discrete symmetry $\mathbb{Z}_{3}$. Vortices are then confined at sufficiently long distances due to a pinning potential for the phase of $\Delta$. Fractionalization can only be observed on length scales much larger than $\Delta_{0}$ yet much smaller than the confining length scale, a function of $\Delta_{0} / t$. This difficulty can be overcome by a small breaking of the point-group symmetry of the honeycomb lattice by assuming anisotropic hopping between nearest-neighbor sites. By continuity, the only effect of such a small anisotropy is to move the Dirac points away from the boundaries of the first Brillouin zone, as is confirmed by an exact computation of the spectrum in Ref. [23]. The Kekulé wave vector $\boldsymbol{G}$ in Eq. (2) is then not anymore commensurate to the reciprocal lattice, integration over the fermions does not produce a pinning potential for the phase of Kekule modulation, and vortices can proliferate as a result of temperature-induced fluctuations (their bare logarithmic interaction is screened for temperatures above the Kosterlitz-Thouless transition temperature).

In summary, we presented a mechanism in graphenelike 2D condensed matter systems that realizes charge fractionalization with TRS. The mechanism is based on an effective low-energy Hamiltonian of the Dirac type with a textured mass with phase twists due to vortices. When the vortices are far apart, each vortex carries a zero-mode responsible for local charge fractionalization. This mechanism is a condensed matter $2+1$ space-time analogue to $3+1$ space-time cosmic strings.

We would like to thank Eduardo Fradkin for illuminating comments about the confinement of vortices for a commensurate Kekulé modulation. This work is supported by the NSF Grant No. DMR-0305482 (C.-Y.H. and C. C.).

[1] R. Jackiw and C. Rebbi, Phys. Rev. D 13, 3398 (1976).

[2] W. P. Su, J. R. Schrieffer, and A. J. Heeger, Phys. Rev. Lett. 42, 1698 (1979).

[3] R. B. Laughlin, Phys. Rev. Lett. 50, 1395 (1983).

[4] B. I. Halperin, Phys. Rev. Lett. 52, 1583 (1984).

[5] D. Arovas, J. R. Schrieffer, and F. Wilczek, Phys. Rev. Lett. 53, 722 (1984).

[6] P. W. Anderson, Science 235, 1196 (1987).

[7] S. A. Kivelson, D. S. Rokhsar, and J. P. Sethna, Phys. Rev. B 35, 8865 (1987).

[8] R. B. Laughlin, Science 242, 525 (1988).

[9] P. R. Wallace, Phys. Rev. 71, 622 (1947).

[10] G. W. Semenoff, Phys. Rev. Lett. 53, 2449 (1984).

[11] K. S. Novoselov, A. K. Geim, S. V. Morozov, D. Jiang, M. I. Katsnelson, I. V. Grigorieva, S. V. Dubonos, and A. A. Firsov, Nature (London) 438, 197 (2005).

[12] E. Witten, Nucl. Phys. B249, 557 (1985).

[13] N. Read and D. Green, Phys. Rev. B 61, 10267 (2000).

[14] R. Jackiw and P. Rossi, Nucl. Phys. B190, 681 (1981).

[15] L.F. Cugliandolo, E. H. Fradkin, and F. A. Schaposnik, Phys. Lett. B 224, 407 (1989).

[16] C. Chamon, Phys. Rev. B 62, 2806 (2000).

[17] We reserve the terminology of particle-hole symmetry to single-particle Hamiltonians of the Bogoliubov-de Gennes type such as in Refs. [13,14].

[18] W. P. Su, J. R. Schrieffer, and A. J. Heeger, Phys. Rev. B 22, 2099 (1980).

[19] J. Goldstone and F. Wilczek, Phys. Rev. Lett. 47, 986 (1981).

[20] M. J. Rice and E. J. Mele, Phys. Rev. Lett. 49, 1455 (1982).

[21] R. Jackiw and G. Semenoff, Phys. Rev. Lett. 50, 439 (1983).

[22] S. Kivelson, Phys. Rev. B 28, 2653 (1983).

[23] Y. Hasegawa, R. Konno, H. Nakano, and M. Kohmoto, Phys. Rev. B 74, 033413 (2006). 